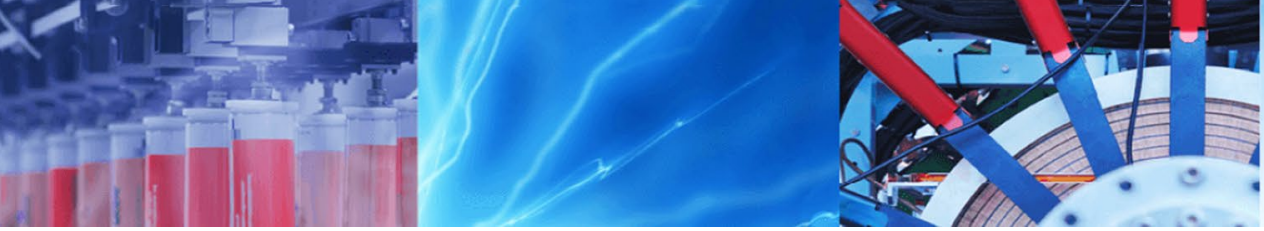

Research Article

\title{
The influence of spiral angle on lift-off speed of face gas seal under start-up
}

\author{
Junjie $\mathrm{Lu}^{1} \mathbb{D} \cdot$ Xuexing Ding $^{2} \cdot$ Wei Zhang $^{1}$
}

(c) Springer Nature Switzerland AG 2019

\begin{abstract}
The lift-off rotating speed of dry gas seal (SG-DGS) is investigated by conducting an experimental test, and using a theoretical model based on the slip-flow effect. The experimental results show that the mixed lubrication regime is transferred to the hydrodynamic lubrication regime at a smaller lift-off rotational speed for a lower spiral angle. On the other hands, the theoretical procedure is implemented to calculate the gas film opening force and the lift-off rotational speed is determined by the minimum contact gas film thickness. The theoretical results show that the smaller spiral angle can offer the greater gas film force and lower lift-off speed. Meanwhile, the experimental data verify the theoretical results well. It can be demonstrated that the spiral angle has a strong effect on the lift-off rotating speed and the theoretical model can calculate the lift-off rotational speed of SG-DGS during start-up process.
\end{abstract}

Keywords Gas film seal · Spiral groove · Start-up · Lift-off speed · Experiment · Theoretical model

\begin{tabular}{|c|c|c|c|}
\hline \multicolumn{2}{|c|}{ List of symbols } & $N$ & Groove number \\
\hline \multicolumn{2}{|c|}{$E \quad$ Half of groove depth $(\mathrm{m})$} & $p$ & Pressure of gas film $(\mathrm{Pa})$ \\
\hline $2 E$ & Groove depth (m) & $p_{\mathrm{i}}$ & Environment pressure $(\mathrm{Pa})$ \\
\hline$f$ & Friction coefficient & $p_{0}$ & Media pressure $(\mathrm{Pa})$ \\
\hline$F$ & Friction force $(\mathrm{N})$ & $P$ & Non-dimensional gas film pressure \\
\hline$F_{c}$ & Closing force $(\mathrm{N})$ & $r$ & Seal ring radius $(\mathrm{m})$ \\
\hline$F_{\mathrm{e}}$ & Force of back side of stationary ring $(\mathrm{N})$ & $R_{a 1}$ & Surface roughness of rotating ring $(\mathrm{m})$ \\
\hline$F_{\mathrm{i}}$ & Interfacial force $(\mathrm{N})$ & $R_{a 2}$ & Surface roughness of static ring $(\mathrm{m})$ \\
\hline$F_{1}$ & Load $(\mathrm{N})$ & $R_{\mathrm{e}}$ & Slip radius $(\mathrm{m})$ \\
\hline$F_{\mathrm{n}}$ & Normal component $(\mathrm{N})$ & $R_{\mathrm{i}}$ & Inner radius $(\mathrm{m})$ \\
\hline$F_{\mathrm{o}}$ & Opening force $(\mathrm{N})$ & $R_{\mathrm{o}}$ & Outer radius $(\mathrm{m})$ \\
\hline$F_{\mathrm{s}}$ & Spring force $(\mathrm{N})$ & $R_{\mathrm{r}}$ & Root radius $(\mathrm{m})$ \\
\hline$F_{\mathrm{T}}$ & Tangential component $(\mathrm{N})$ & $\mathrm{R}$ & Gas constant \\
\hline$h$ & Lubrication film thickness (m) & $t$ & Time (s) \\
\hline$h_{\mathrm{c}}$ & Minimum contact film thickness (m) & $u$ & Circumferential velocity $(\mathrm{m} / \mathrm{s})$ \\
\hline $\mathrm{H}$ & Non-dimensional lubrication film thickness & $U_{0}$ & Linear velocity $(\mathrm{m} / \mathrm{s})$ \\
\hline Kn & Knudsen number & $v$ & Radial velocity (m/s) \\
\hline I & Molecular free path (m) & $w$ & Gas velocity component $(\mathrm{m} / \mathrm{s})$ \\
\hline$L$ & Equivalent arm length $(\mathrm{m})$ & $x$ & Component of external force $(\mathrm{N})$ \\
\hline$M$ & Friction torque (N.m) & $Y$ & Component of external force $(\mathrm{N})$ \\
\hline$n_{\mathrm{r}}$ & Rotational speed (r/min) & $z$ & Direction of gas film thickness \\
\hline
\end{tabular}

Junjie Lu, loveljj4566@163.com | 'Zhejiang University Ningbo Institute of Technology, Ningbo, China. ${ }^{2}$ College of Petrochemical Engineering, Lanzhou University of Technology, Lanzhou, China.

SN Applied Sciences (2019) 1:1068 | https://doi.org/10.1007/s42452-019-1103-2

Received: 11 February 2019 / Accepted: 14 August 2019 / Published online: 21 August 2019 
Z Component of external force $(\mathrm{N})$

a Spiral angle $\left({ }^{\circ}\right)$

$\beta \quad$ Complementary angle of spiral angle $\left(^{\circ}\right)$

$\beta_{0} \quad$ Groove coefficient

$\delta \quad$ Normal film thickness $(m)$

$\varepsilon \quad$ Small parameter of iterative perturbation

$\zeta \quad$ Non-dimensional radius

$\zeta_{0} \quad$ Non-dimensional outer radius

$\eta_{\mathrm{i}} \quad$ Parameters of gas film force $(i=1,2)$

$\theta \quad$ Polar coordinate

$\mu \quad$ Dynamic viscosity of gas (Pa.s)

$\rho \quad$ Gas density $\left(\mathrm{kg} / \mathrm{m}^{3}\right)$

$\sigma \quad$ Contact pressure in a unite area $\left(\mathrm{N} / \mathrm{m}^{2}\right)$

$\sigma_{\mathrm{s}} \quad$ Standard deviation combined roughness of both surfaces

$\sigma_{\mathrm{v}} \quad$ Regulation coefficient about tangential component

$\varphi \quad$ Non-dimensional angle

$\chi \quad$ Compressible correction coefficient under slip boundary condition

$\omega \quad$ Equivalent spiral angle

$\wedge$ Compressible coefficient

$\nabla \quad$ Laplace operator

\section{Integration constant}

$\begin{array}{ll}A_{i} & (i \text { is } 1,2,3) \\ B_{i} & (i \text { is } 1,2,3) \\ C_{i j} & (i \text { is } 1,2 \text { and } j \text { is } 0,1) \\ C_{i j}^{\prime} & (i \text { is } 1,2 \text { and } j \text { is } 0,1)\end{array}$

\section{Introduction}

A spiral groove dry gas seal (SG-DGS) is a type of non-contacting mechanical face seal widely used in turbomachinery system [1, 2]. Spiral grooves with a depth of several micrometres are machined into a rotating ring, enabling the generation of hydrodynamic effects and providing a "lift-off force" (opening force) that completely separates the rotating ring and static ring. Several studies have been conducted to analyse the behaviour of a gas seal in gas film lubrication performance and seal rings tribology.

In the field of lubrication performance, Miller et al. [3] developed a novel semi-analytical formulation to conduct a linearized dynamic analysis of spiral-grooved mechanical gas face seals. In addition, Xiao et al. [4] proposed a one-dimensional radial discretization method to study the radial variations of different parameters of face seals and found that the proposed model provided better accuracy. Zhang et al. [5] and Ruan [6] researched a representative spiral groove seal and a micro gas journal bearing by a modified Reynolds Equation that considered slip flow effects at slow speed and low-pressure conditions. It presented a numerical model of the thermos-hydrodynamic of mechanical face seals and gas face seals to anlyse the effect of thermal distortion on seal performance and design parameters under different rotating speeds and different pressures [7]. Ding et al. [8] used the experimental tests and theoretical calculations to observe the temperature distribution of a gas film in a dry gas seal. With respect to face tribology, it was presented a transient dynamic analysis of mechanical seals with respect to asperity contact, face deformation, and the separation of contacting seals [9]. An experimental method and procedure was illustrated to study on the friction and wear of carbon-graphite mechanical face seals. Goilkar et al. [10] designed and developed the experimental setup to simulate the actual industrial environment encountered by a mechanical seal. Wang et al. [11] tested the tribological performance of a specimen with three sealed fluid conditions. The results indicated that the coatings could endure a cryogenic temperature, while the friction coefficients decreased with the increase in the contact load. Qiu et al. [12] designed a series of experiments to study the tribological behaviour of spiral groove thrust bearings and developed a theoretical model to study the frictional characteristics of spiral grooves in both the hydrodynamic regime and the mixed lubrication regime. A novel variable ovoid non-smooth structure was proposed including the radius, semimajor, and depth and the flow characteristics were calculated. Results of skin friction reduction were obtained by orthogonal testing [13].

The influence of the spiral angle on the bearing capacity of bearings was investigated by Whipple [14] and then further completed research was done by Muijderman [15]. However, only a few studies have examined the starting/stopping process of face seals. Huang et al. [16] investigated the behaviour of a double dry gas seal by acoustic emission testing under starting and stopping conditions. Salant et al. [17] utilized the Duhamel's model of a mechanical seal and determined Duhamel's auxiliary function. The results predicted the performance of a mechanical seal during start-up and shutdown. Liu et al. [18] proposed a three-dimensional thermal elastic hydrodynamic model of a wavy-tilt-dam mechanical seal and studied seal mechanisms under quasi-start-up and steady-state conditions. During the start-up process, low axial stiffness which is resulted from little gas film opening force can lead to the contact and even worst scratches of sealing rings, sometimes causing great wear of sealing rings so that seal failure.

In the current study, an experiment is conducted on SGDGS with different spiral groove angles to evaluate their tribological behaviour and thereby determining the variation in lift-off rotational speed during start-up. Additionally, a theoretical model of SG-DGS is established based on the modified Reynolds Equation and the slip-flow effect, 
and then approximate values for the lift-off rotational speed are obtained. The calculation results are presented to evaluate the lift-off rotational speed of SG-DGS and the relationship between the geometric parameters of spiral grooves and the lift-off rotational speed, as verified by the experimental results.

\section{Experiment}

\subsection{Specimens}

During startup, stable operation and shut-down of dry gas seal, the gap between the static ring and the rotating ring forms a gas film, and the static and dynamic ring constitutes a pair of friction pairs. The experimental specimens in this study are static and dynamic (rotating) rings that can be called as RR (rotating ring) and SR (stationary ring). The RRs are made of SiC, and the SRs are made of graphite. Spiral grooves are machined into the surface of the RRs. In this test, we want to observe the influence of different spiral angles on the rotational speed. Therefore, a rotating ring corresponds to a spiral angle. The geometric and physical parameters of the specimens are listed in Table 1. According to different spiral angles of the rotating ring, four sets of test specimens are provided: each set of test specimens is composed of a rotating ring and a static ring, forming a pair of friction pairs.

The surface of RR is grooved. In the current study, a fibre laser marking (engraving) machine is used to engrave spiral grooves on the rotary ring. The pattern is transferred into the laser marking machine, and the machine carved spiral grooves in the outer surface of RR. The spiral groove depth and roughness can be controlled by the number of times the laser passes the region of a spiral groove and the power of the laser [12]. Figure 1 shows the technological process of specific groove and the 3D surface profile of the specimen by ZeGage.

\subsection{Experiment equipment}

The experiment is performed on an HDM-2 end-face friction and wear experiment machine, as shown in Fig. 2 . The test machine consists of a host computer and a computer measurement and control system. The main machine consists of a main shaft, upper and lower test piece clamps, upper and lower test piece boxes, load device and drive device.

The rotating upper specimen and the static lower specimen is contacted by the HDM- 2 machine. In this study, the upper specimen is the rotating ring and the lower specimen is the static ring. The speed sensors are installed in shaft of transmission equipment and the pressure sensors are installed the end of hydraulic loading equipment.

The sensors signal is transformed as physical signal, after which the digital signal is realized by data acquisition. The digital signal is transmitted to a computer and displayed in output equipment. As the friction coefficient cannot be directly measured with a sensor, the friction torque is directly measured in the test, and then the friction torque is converted into the friction coefficient. The torque sensor is connected to the lower specimen, the friction torque measured is transferred to the computer for reading, and the friction torque is converted into the friction coefficient based on the relation between them. The contact of rotating ring and static ring produce friction force. Therefore, a force transducer is installed in the assembly sleeve of the sealing ring, and the torque can be calculated based on the length of force transducer and
Table 1 Geometric parameters of the specimens

\begin{tabular}{|c|c|c|c|c|c|c|c|}
\hline \multirow[t]{3}{*}{ Specimens \# } & \multicolumn{6}{|c|}{ Geometry } & \multirow{3}{*}{$\begin{array}{l}\text { Ring surface } \\
\text { Roughness (m) }\end{array}$} \\
\hline & \multicolumn{3}{|c|}{ Groove parameters } & \multicolumn{3}{|c|}{ Ring radius (m) } & \\
\hline & Angle & Depth (m) & Number & Inner & Outer & Slip & \\
\hline \multicolumn{8}{|l|}{ Group 1} \\
\hline $\mathrm{RR} 12^{\circ}-1$ & $12^{\circ}$ & $0.1 \times 10^{-4}$ & 16 & 0.031 & 0.041 & & $2.12 \times 10^{-8}$ \\
\hline SR-1 & & & & 0.031 & 0.041 & 0.033 & $1.6 \times 10^{-7}$ \\
\hline \multicolumn{8}{|l|}{ Group 2} \\
\hline $\mathrm{RR} 14^{\circ}-2$ & $14^{\circ}$ & $0.1 \times 10^{-4}$ & 16 & 0.031 & 0.041 & & $2.15 \times 10^{-8}$ \\
\hline SR-2 & & & & 0.031 & 0.041 & 0.033 & $1.54 \times 10^{-7}$ \\
\hline \multicolumn{8}{|l|}{ Group 3} \\
\hline RR16 ${ }^{\circ}-3$ & $16^{\circ}$ & $0.1 \times 10^{-4}$ & 16 & 0.031 & 0.041 & & $2.13 \times 10^{-8}$ \\
\hline SR-3 & & & & 0.031 & 0.041 & 0.033 & $1.56 \times 10^{-7}$ \\
\hline \multicolumn{8}{|l|}{ Group 4} \\
\hline RR18 ${ }^{\circ}-4$ & $18^{\circ}$ & $0.1 \times 10^{-4}$ & 16 & 0.031 & 0.041 & & $1.56 \times 10^{-8}$ \\
\hline SR-4 & & & & 0.031 & 0.041 & 0.033 & $1.57 \times 10^{-7}$ \\
\hline
\end{tabular}




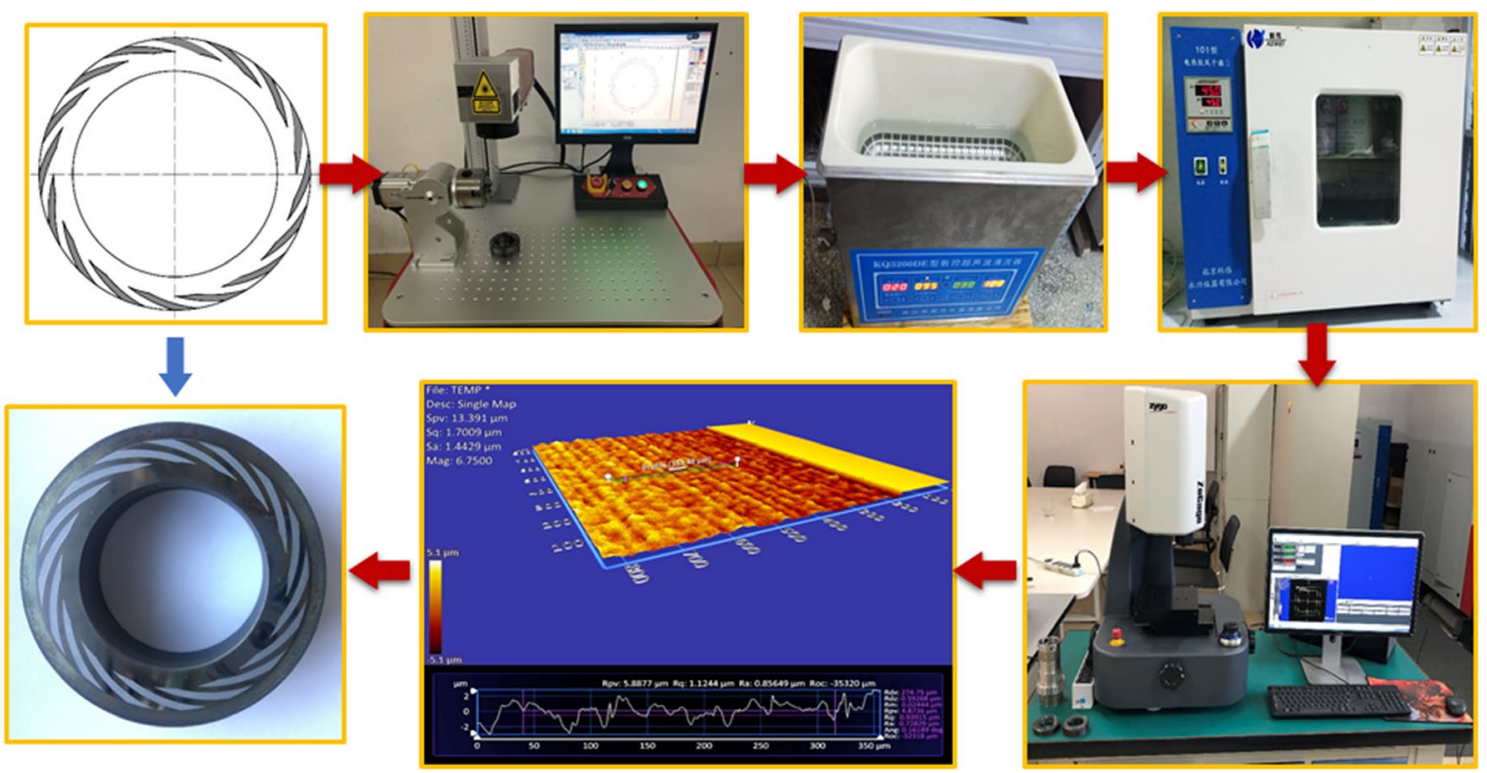

Fig. 1 Machining and testing of the rotating ring

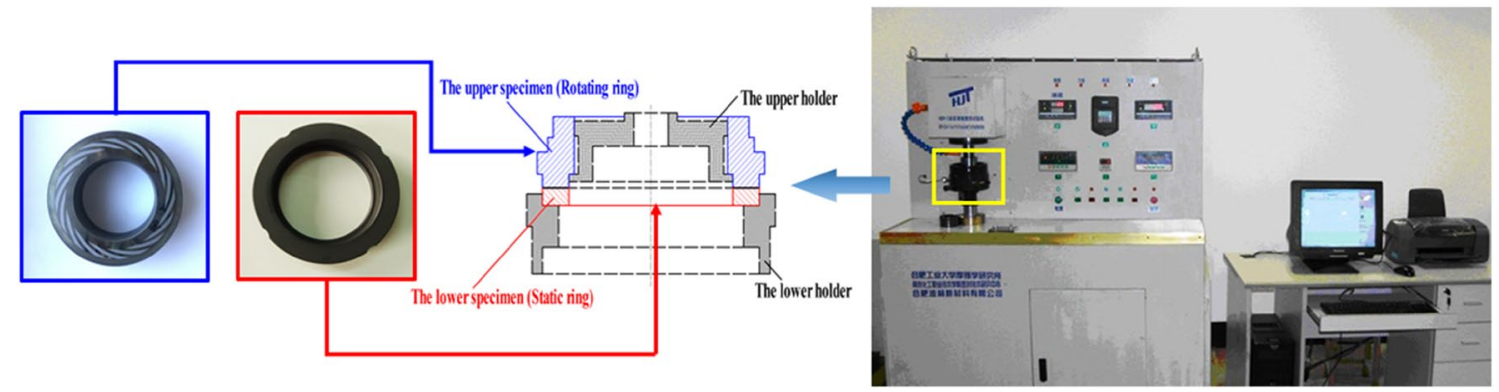

Fig. 2 HDM-2 end-face friction and wear experiment machine

the friction force. The conversion relation between friction torque and friction coefficient is as follows:

$M=F L=\int_{r}^{r} \pi 2 \mu \sigma r^{2} \mathrm{~d} r=\frac{2}{3} \pi \mu \sigma\left(R_{o}^{3}-R_{i}^{3}\right)$

where $M$ is the friction torque; $F$ is the friction force; $L$ is the equivalent arm length, $L=0.08 \mathrm{~m} ; \sigma$ is the contact pressure in a unit area.

$\sigma=F_{l} /\left[\pi\left(R_{o}^{2}-R_{i}^{2}\right)\right]$

where $R_{\mathrm{i}}$ is the inner radius of the upper specimen; $R_{\mathrm{o}}$ is the outer radius of the upper specimen.

Therefore, the friction coefficient can be calculated by torque and size of sealing end face:

$$
\begin{aligned}
f & =3 F L /\left[2 \pi \sigma\left(R_{o}^{3}-R_{i}^{3}\right)\right] \\
& =3\left(R_{o}^{2}-R_{i}^{2}\right) F L /\left[2\left(R_{o}^{3}-R_{i}^{3}\right) F_{l}\right] \\
& =\frac{3}{2} \frac{R_{o}+R_{i}}{R_{o}^{2}+R_{o} R_{i}+R_{i}^{2}} \frac{M}{F_{l}}
\end{aligned}
$$

where $F_{1}$ is the load, and $f$ is the friction coefficient.

\subsection{Experiment procedure}

In this test, the pressure is set at $0.12 \mathrm{MPa}$. According to the pressure and Eq. (6), the load is calculated ( $226 \mathrm{~N})$. The pressure is medium pressure of dry gas seal under start-up condition. The rotating speed is designed and increase by $50 \mathrm{r} / \mathrm{min}$ at a time during $50-800 \mathrm{r} / \mathrm{min}$. The time spent at each discrete rotating speed is programmed to $3 \mathrm{~min}$ so that the happens in temperature will be little.

The detailed experimental steps are as follows:

1. Group 1 is installed on the HDM-2 end-face friction and wear experiment machine, $\mathrm{RR} 12^{\circ}-1$ is as the upper test specimen and SR-1 is as the lower test specimens.

2. The final installation location and the mutual assembly location of the upper and lower test specimens are marked. 
3. The contact load of the friction pair is adjusted to $226 \mathrm{~N}$, the lower specimen is lifted slowly and contacted with the upper specimen.

4. When the contact load of friction pair is reached $226 \mathrm{~N}$, the load device is stopped.

5. The testing machine starts to run and the rotating speed is raised in segment of $50 \mathrm{r} / \mathrm{min}$ from 50 up to $800 \mathrm{r} / \mathrm{min}$.

6. The relevant data is recorded during the test.

7. After the machine is stopped, the first group of specimens is removed.

8. Groups 2,3 and 4 are tested and followed the steps (1)-(7).

\section{Experiment results}

Figure 3 present the friction coefficient of the specimens with the rotational speed. As shown in Fig. 3, the friction coefficient decreases gradually to a minimum value and then increases.

The curve of the friction coefficient illustrate the operating characteristics of SG-DGS during start-up. The upper and lower specimens are in close contact before running. When the upper specimen starts to rotate, the rotating speed is very low, thus, no pressure built up in the lubrication, and $100 \%$ of the load is carried by the asperities in the contact area. Therefore, the friction coefficient reaches their maximum values first, as shown in the Fig. 3. In addition, under the wedging and throttling effects on the spiral grooves, hydrodynamic pressure builds up in the lubrication as the rotating speed increase. However, the gas film is too thin to separate the surfaces of the sealing rings. The load is carried by a combination of the hydrodynamic pressure and the contact pressure between the asperities

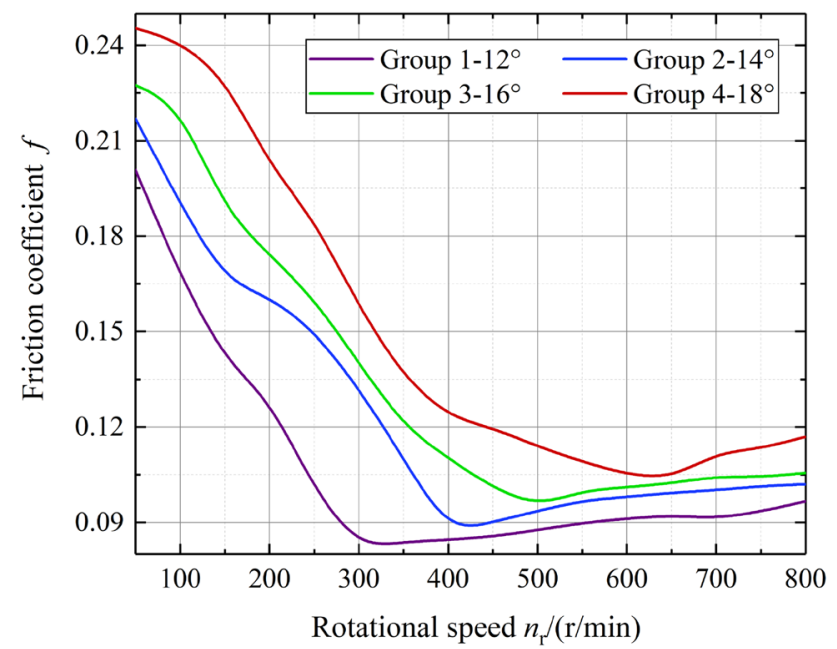

Fig. 3 Friction coefficient with rotational speed for Groups 1-4 of both surfaces. Thus, it is the mixed lubrication that is the intermediate region between the boundary lubrication and hydrodynamic lubrication. Therefore, as shown in Fig. 3, the friction coefficient begins to decrease appreciably. On the other hand, when the lubricated regime of SGDGS is the mixed lubrication, the RR and SR of the SG-DGS are not completely separated. The abrasion between the sealing faces increase with the rotating speed. The abrasive grains on the surface are mostly composed of graphite from the SR, which shows good lubrication performance (the lower hardness of the SR makes abrasion more likely to occur). Therefore, the abrasive particles act as a lubricant between the sealing faces and reduce the friction, which is the embodiment of the self-lubrication principle of the SR. As the rotating speed increases continuously, the hydrodynamic pressure increases in the spiral grooves such that the surfaces of the sealing rings are completely separated by a gas film. The lubrication condition is hydrodynamic lubrication or full-film lubrication. The friction force is caused by viscous shear in the gas molecular layer, which is affected by the shear stress and shear area. As the rotational speed increase, the hydrodynamic pressure and shear stress increases. Therefore, the friction force between the surfaces of the sealing rings increase, causing the friction coefficient to increase. But the friction coefficient is increased slowly because of the low gas viscosity.

To explain the variation of the friction coefficient, it is useful to evaluate the relationship between the different lubrication regimes and friction coefficients and examine the behaviour of different lubrication regimes by applying the Stribeck curve as shown in Fig. 4. The Stribeck curve could be a reference to judge the lubrication behaviour. It is observed that the minimum value of the friction coefficient as the demarcation between full-film (hydrodynamic) lubrication and mixed lubrication (some solid asperity interactions). The rotational speed corresponding to the demarcation point is the transition speed. Thus, each of the minimum friction coefficients in Fig. 3 is considered the transition point between the mixed lubrication regime and the hydrodynamic lubrication regime. The lift-off rotational speed is therefore the rotating speed that corresponds to the transition point. The Groups 1, 2, 3, and 4 with the spiral angles of $12^{\circ}, 14^{\circ}, 16^{\circ}$ and $18^{\circ}$ is obtained the lift-off rotational speed $300 \mathrm{r} / \mathrm{min}, 400 \mathrm{r} / \mathrm{min}, 500 \mathrm{r} / \mathrm{min}$ and $650 \mathrm{r} / \mathrm{min}$ respectively.

An interesting characteristic of Fig. 3 is that an increase in the spiral angle results in a higher lift-off rotating speed. This finding indicates that a lower spiral angle can provide a greater gas film force and enter full-film lubrication at a smaller rotational speed, which enables the SG-DGS to reach lift-off more easily and reduces wear. When the rotating ring begins to rotate, the gas moves along with the spiral grooves. The gas film force is generated by the spiral 


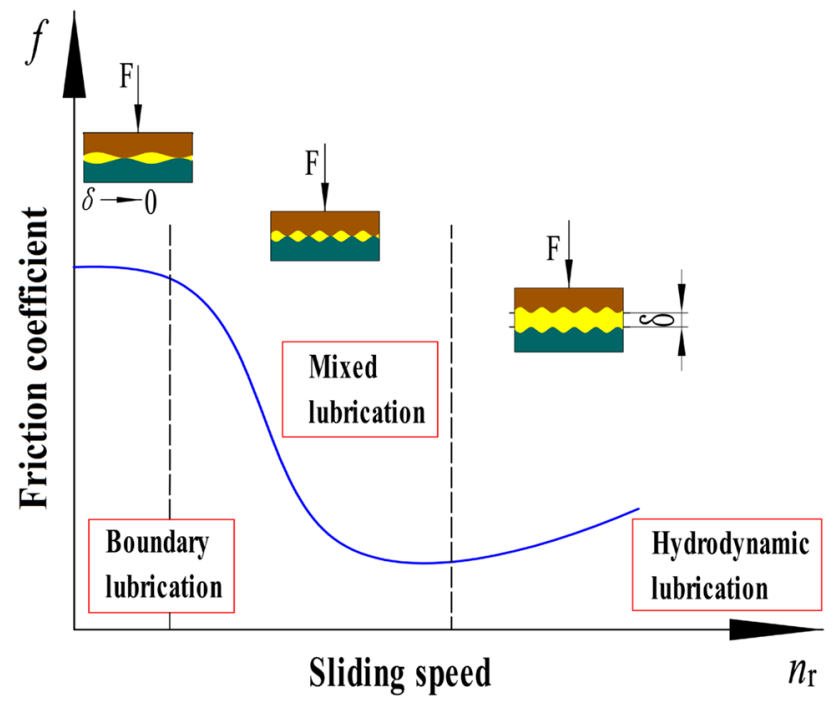

Fig. 4 Stribeck curve

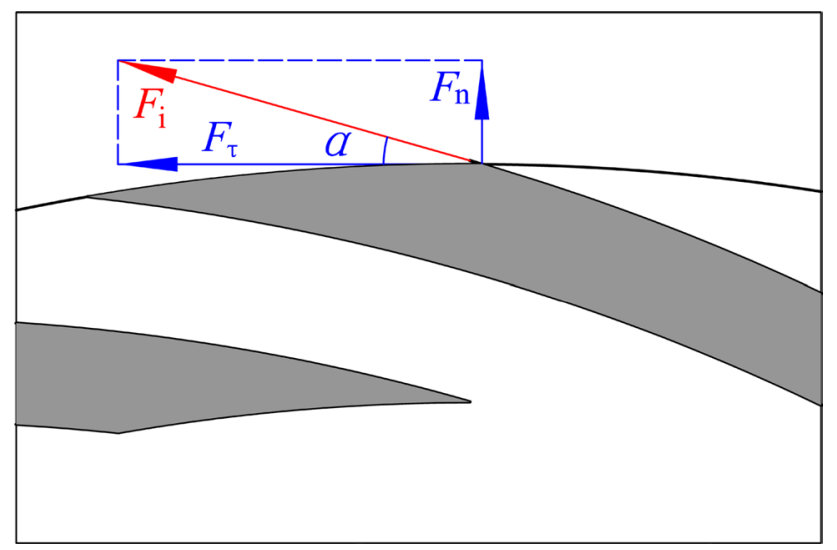

Fig. 5 Force simplified model with spiral groove grooves. What's more, the gas film force can decompose as the radial component and tangential component, as shown in Fig. 5, where, $F_{\mathrm{i}}$ is the interfacial force, $a$ is the spiral angle, $F_{\mathrm{n}}$ is the radial component $\left(F_{\mathrm{n}}=F_{\mathrm{i}} \cdot \sin \alpha\right)$ and $F_{\mathrm{T}}$ is the tangential component $\left(F_{\mathrm{T}}=F_{\mathrm{i}} \cdot \cos \alpha\right)$. The force of tangential component can help to improve the pumping and gas compression effects of SG-DGS. Meanwhile, according to the specimens with different spiral angles, $F_{\mathrm{T}}\left(a=12^{\circ}\right)>F_{\mathrm{T}}\left(a=14^{\circ}\right)>F_{\mathrm{T}}\left(a=16^{\circ}\right)>F_{\mathrm{T}}\left(a=18^{\circ}\right)$. Thus, the specimen with the spiral angle $12^{\circ}$ can provide the better load-carrying capacity and the lower lift-off rotational speed than other specimens.

\section{The theoretical model of SG-DGS}

\subsection{The governing equations}

Schematics of a spiral groove seal rotating ring is established as depicted in Fig. 6 . The seal gas is pump into the seal housing. When gas is injected into the spiral groove, the wedging and pumping effects cause the gas to become gradually compressed and the pressure to increase, providing an opening force that separates the surfaces. $a$ is the spiral angle, $n_{r}$ is the rotating speed, $R_{i}$ is the inner radius, $R_{o}$ is the outer radius, $p_{i}$ is the environmental pressure, and $p_{o}$ is the medium pressure. The logarithmic spiral is considered by the relation as follows:

$r=R_{r} e^{\theta \cdot \tan (\alpha)}$

where $r$ is the seal ring radius and $\theta$ is a polar coordinate.

The SG-DGS is considered to operate at low speed and low pressure during start-up, and the gas film thickness is about micrometres, placing it on the micro-scale. The
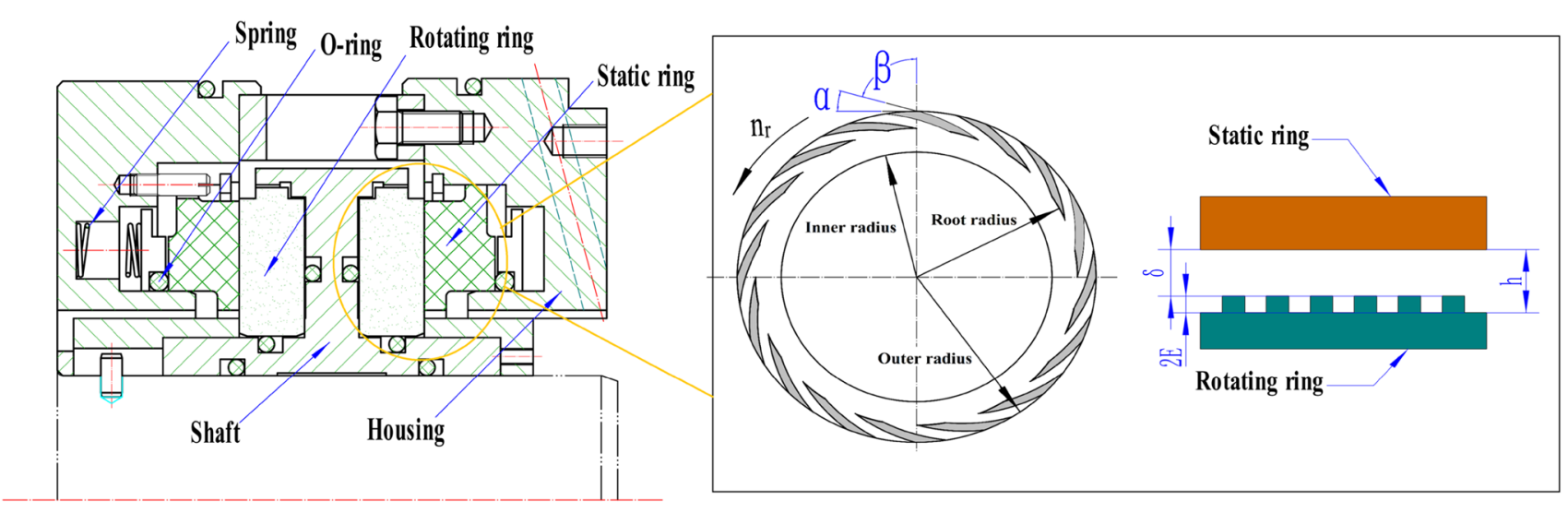

Fig. 6 A schematic of SG-DGS 
gas film is described to flow in a narrow gap. The Knudsen number $(K n=l / h, l$ is the gas molecular free path, $h$ is the lubrication film thickness) is used as an indicator of gas rarefaction. The gas flow varies from continuum flow, slip flow, and transition flow to free molecular flow as $K n$ varies from zero to infinity. The effect of gas rarefaction is widely discussed in the field of thin film gas lubrication, which is the regime that occurs between mixed lubrication and hydrodynamic lubrication. Based on the lubrication film thickness and air molecular free path of the SG-DGS under start-up, the value of the Knudsen number is less than 0.1 . When the Knudsen number is during $0.1-0.001$, this suggests that the slip velocity of the interface between the gas film and solid surface is generated, as proposed Beskok et al. [19]. Thus, the boundary conditions on the solid surface is confirmed. According to the Ref. [6], the second-order slip-flow model of the boundary conditions is modified so that it could be applied to increase calculational stability. Based on the characteristics of a compressible fluid flowing in the narrow gap that occurs during the start-up process and by considering the slip velocity and stress continuity, the following basic assumptions are made:

1. Equal temperature and equal viscosity.

2. The gas is an ideal gas.

3. The gas flow is considered laminar flow.

4. The inertial and volume forces of the gas are neglected.

5. The gas film between the rotating ring and stationary ring is considered a Newtonian fluid.

The gas film thickness of the SG-DGS is determined by the forces acting on the sealing rings, and the axial force balance is written as

$F_{c}=F_{o}$

where

$F_{c}=p_{o} \pi\left(R_{o}^{2}-R_{e}^{2}\right)+F_{s}$

and

$F_{o}=2 \pi \int_{R_{i}}^{R_{o}} r p d r$

where $F_{c}$ is the closing force, $F_{o}$ is the opening force, $F_{s}$ is the spring force, $p_{\mathrm{o}}$ is the media pressure of the SG-DGS, $R_{\mathrm{o}}$ is the outer radius, $R_{\mathrm{i}}$ is the inner radius and $R_{\mathrm{e}}$ is the slipping radius.

Burgdorfer [20] derives the Reynolds equation that is isothermal solid wall considering the slip condition under the following $\mathrm{N}-\mathrm{S}$ equation and the slip boundary condition.The Navier-Stokes $(\mathrm{N}-\mathrm{S})$ equations are expressed as $\left\{\begin{array}{l}\rho(d u / d t)=-(\partial p / \partial x)+\rho X+\mu \nabla^{2} u \\ \rho(d v / d t)=-(\partial p / \partial y)+\rho Y+\mu \nabla^{2} v \\ \rho(d w / d t)=-(\partial p / \partial z)+\rho Z+\mu \nabla^{2} w\end{array}\right.$

where $\nabla$ is the Laplace operator; $\rho$ is fluid density; $p$ is pressure; $u, v$, and $w$ are velocity components; $X, Y$, and $Z$ are the components of the external force; and $\mu$ is dynamic viscosity.

Gas movement develops between the rotating and stationary rings in the spiral groove dry gas seal. Thus, the gas movement is similar to the two-dimensional motion between parallel plates, and the $\mathrm{N}-\mathrm{S}$ equations in Cartesian coordinates can be simplified as

$\partial p / \partial x=\mu\left(\partial^{2} u / \partial z^{2}\right)$

$\partial p / \partial y=\mu\left(\partial^{2} v / \partial z^{2}\right)$

where $z$ is the direction of the gas film thickness.

According to the Ref. [6] and [19], when slip occurs between the upper and lower plates, the slip boundary conditions are $z=0$ :

$u=U_{0}+l^{\prime}(\partial u / \partial z)-\left(l^{\prime 2} / 2\right)\left(\partial^{2} u / \partial z^{2}\right)$

$v=I^{\prime}(\partial v / \partial z)-\left(I^{\prime 2} / 2\right)\left(\partial^{2} v / \partial z^{2}\right)$

$z=h$ :

$u=-l^{\prime}(\partial u / \partial z)-\left(I^{2} / 2\right)\left(\partial^{2} u / \partial z^{2}\right)$

$v=-l^{\prime}(\partial v / \partial z)-\left(I^{\prime 2} / 2\right)\left(\partial^{2} v / \partial z^{2}\right)$

where $U_{0}=2 \pi n_{\mathrm{r}} R_{\mathrm{i}}, U_{0}$ is the linear velocity, $n_{\mathrm{r}}$ is the rotational speed, $R_{\mathrm{i}}$ is the inner radius of the sealing ring, $I^{\prime}=I\left(2-\sigma_{\mathrm{v}}\right) / \sigma_{\mathrm{v}} /$ is the molecular free path, and $\sigma_{\mathrm{v}}$ is the regulation coefficient about tangential component.

The circumferential and radial velocities of the gas are determined as follows:

The continuous double integral method is applied to Eq. (9b),

$v=(1 / 2 \mu)(\partial p / \partial y) z^{2}+A z+B$

where $A$ and $B$ are variable notations.

Combined with the boundary conditions described by Eqs. (10b) and (11b),

$A=\left(-2 l^{\prime} / h\right)(\partial v / \partial z)-(h / 2 \mu)(\partial p / \partial y)$,

$B=I^{\prime}(\partial v / \partial z)-\left(I^{2} / 2\right)\left(\partial^{2} v / \partial z^{2}\right)$

Furthermore,

$\partial v / \partial z=[(2 z-h) / 2 \mu]\left[h /\left(h+2 l^{\prime}\right)\right](\partial p / \partial y)$,

$\partial^{2} v / \partial z^{2}=(1 / \mu)(\partial p / \partial y)$

The radial velocity of a gas film can be expressed as 
$v=\left[\left(z^{2}-h z-h l^{\prime}\right) / 2 \mu\right](\partial p / \partial y)$

$$
+\left[\left(h l^{\prime}-2 z l^{\prime}\right)(2 z-h) /\left(2 \mu h+4 \mu l^{\prime}\right)\right](\partial p / \partial y)
$$

According to the same method, the boundary conditions (10a) and (11a) are substituted into Eq. (9a), and the circumferential velocity is expressed as

$$
\begin{aligned}
u= & {\left[\left(z^{2}-h z-h l^{\prime}\right) / 2 \mu\right](\partial p / \partial x) } \\
& +\left[\left(h l^{\prime}-2 z l^{\prime}\right)(2 z-h) /\left(2 \mu h+4 \mu l^{\prime}\right)\right](\partial p / \partial x) \\
& -U_{0}\left(h l^{\prime}-2 z l^{\prime}\right) /\left(h^{2}+2 h l^{\prime}\right)+U_{0}(1-z / h)
\end{aligned}
$$

The continuity equation is expressed as

$(\partial \rho / \partial t)+(\partial \rho u / \partial x)+(\partial \rho v / \partial y)+(\partial \rho w / \partial z)=0$

when $w=0$, Eq. (15) becomes

$(\partial \rho / \partial t)+(\partial \rho u / \partial x)+(\partial \rho v / \partial y)=0$

where $t$ is time and $u, v$, and $w$ are velocity components.

According to Eq. (16), the integral equation is written as

$\int_{0}^{h}[(\partial \rho u / \partial x)+(\partial \rho v / \partial y)] d z+(\partial \rho h / \partial t)=0$

Equations (13) and (14) are substituted into Eq. (17) and solved as follows:

$$
\begin{aligned}
& \rho\left[\left(\partial \int_{0}^{h} u d z\right) /(\partial x)\right] \\
& +\rho\left[\left(\partial \int_{0}^{h} v d z\right) /(\partial y)\right]+\partial(\rho h) / \partial t=0
\end{aligned}
$$

Thus,

$$
\begin{aligned}
\rho[ & {\left.\left[\left(\partial \int_{0}^{h} v d z\right)\right) / \partial y\right] } \\
& \left.=\rho \partial\left\{\int_{0}^{h}\left[\left(z^{2}-h z-h l^{\prime}\right)\left(h+2 l^{\prime}\right)+\left(l^{\prime}-2 z l^{\prime}\right)(2 z-h)\right] /\left(2 \mu h+4 \mu l^{\prime}\right)(\partial p / \partial y)\right] d z / \partial y\right\} \\
& =-\rho \partial\left[\left(h^{3}+6 h^{2} l^{\prime}\right) /(12 \mu)(\partial p / \partial y)\right] / \partial y
\end{aligned}
$$

The two-dimensional steady Reynolds equation considering slip boundary conditions is expressed as

$$
\begin{gathered}
\partial\left\{\left(p h^{3}\right)\left[1+6\left(l^{\prime} / h\right)\right] /(12 \mu)(\partial p / \partial x)\right\} / \partial x+\partial\left\{\left(p h^{3}\right)\right. \\
\left.\left[1+6\left(I^{\prime} / h\right)\right] /(12 \mu)(\partial p / \partial y)\right\} / \partial y \\
=(1 / 2) U_{0}[\partial(p h) / \partial x]
\end{gathered}
$$

By substituting $k n=l^{\prime} / h$ into Eq. (22), the Reynolds equation is written as

$$
\begin{aligned}
& \partial\left[\left(p h^{3} / \mu\right)(1+6 k n)(\partial p / \partial x)\right] / \partial x \\
& +\partial\left[\left(p h^{3} / \mu\right)(1+6 k n)(\partial p / \partial y)\right] / \partial y \\
& \quad=6 U_{0}[\partial(p h) / \partial x]
\end{aligned}
$$

where $k n$ is the Knudsen number, and $k n=l^{\prime} / h$; $h$ is the lubrication film thickness.

The non-dimensional method with $\mathrm{P}=p / p_{\mathrm{i}}$ and $\mathrm{H}=h$ / $(E+\delta)$ is applied to Eq. (23):

$$
\begin{aligned}
& {[1 /(1+6 k n)]\left[(\delta+E)^{2} p_{i} / \mu\right]\left\{\partial\left[\mathrm{PH}^{3}(\partial \mathrm{P} / \partial x)\right] / \partial x\right\}} \\
& \quad+[1 /(1+6 k n)]\left[(\delta+E)^{2} p_{i} / \mu\right] \\
& \left\{\partial\left[\mathrm{PH}^{3}(\partial \mathrm{P} / \partial y)\right] / \partial y\right\}=6 U_{0}[\partial(\mathrm{PH}) / \partial x]
\end{aligned}
$$

Equation (24) is transformed after using polar coordinates, with $\zeta=y / R_{\mathrm{i}}$ and $\varphi=x / R_{\mathrm{i}}$, and simplified as follows:

$$
\partial\left[\mathrm{PH}^{3}(\partial \mathrm{P} / \partial \varphi)\right] / \partial \varphi+\partial\left[\mathrm{PH}^{3}(\partial \mathrm{P} / \partial \zeta)\right] / \partial \zeta=\chi[\partial(\mathrm{PH}) / \partial \varphi]
$$

where

$$
\begin{aligned}
& \chi=\Lambda /(1+6 k n), \\
& \Lambda=\left[12 \pi \mu n_{r} R_{i}^{2} / p_{i}(\delta+E)^{2}\right],
\end{aligned}
$$

Similarly,

$$
\begin{aligned}
& \rho\left[\left(\partial \int_{0}^{h} u d z\right) / \partial x\right] \\
& \quad=-\rho \partial\left[\left(h^{3}+6 h^{2} l^{\prime}\right) /(12 \mu)(\partial p / \partial x)\right] / \partial x+\rho\left[\partial\left(U_{0} h\right) / \partial x\right] / 2
\end{aligned}
$$

The equation of the gas state can be expressed as $p=\rho R T$

where $\mathrm{R}$ is the gas constant and $T$ is temperature. where $\chi$ is the compressible correction coefficient under slip boundary conditions, $\Lambda$ is the compressible coefficient, $\mathrm{P}$ is the non-dimensional gas film pressure, $\mathrm{H}$ is the non-dimensional lubrication film thickness, $\varphi$ is the nondimensional polar angle, $\zeta$ is the non-dimensional polar radius, $\delta$ is the gas film thickness, $E$ is half of the groove depth, and $p_{\mathrm{i}}$ is the environmental pressure.

Gas film pressure is generated between the rotational rings. Thus, the outer and inner radii of the rotational rings are considered the media and environmental pressure boundaries. The boundary conditions in dimensionless form are 
$\left\{\begin{array}{l}\text { when } \zeta=R_{i} / R_{i}=1, \mathrm{P}=p_{i} / p_{i}=1 \\ \text { when } \zeta=R_{o} / R_{i}, \quad \mathrm{P}=p_{o} / p_{i}\end{array}\right.$

The key to solve Eqs. (25) and (26) is the PH linear method and iteration method. The pressure and the film thickness is considered the variables with respect to each other [21]. The nonlinear partial differential equation can be converted to a linear partial differential equation that it is expected in the PH linear method. And the roughly analytical solution to the distribution of gas pressures can be calculated as follows:

$\mathrm{P}=1+\eta\left(\eta_{1(\zeta)} \cos w+\eta_{2(\zeta)} \sin w\right) / \mathrm{H}$

Equation (27) is transformed into a dimensional equation,

$p=p_{i}\left[h+\eta\left(\eta_{1(\zeta)} \cos w+\eta_{2(\zeta)} \sin w\right)(\delta+E)\right] / h$

The opening force of the gas film is considered to be

$F_{o}=2 \pi \int_{R_{i}}^{R_{o}} r p d r$

By substituting Eq. (28) into Eq. (29), the equation for the opening force is expressed as

$$
\begin{aligned}
F_{o}= & {\left[\frac{p_{i}}{1-\varepsilon_{z} \cos \varphi-(E \cos \omega)(E+\delta)}\right.} \\
& +\frac{\left(E p_{i}\right)(E+\delta)\left(\eta_{1(\zeta)} \cos \omega+\eta_{2(\zeta)} \cos \omega\right)}{1-\varepsilon_{z} \cos \varphi-(E \cos \omega)(E+\delta)} \\
& \left.-\frac{3}{2} \beta_{0} E^{2}(E+\delta)^{2} \eta_{2(\zeta)}\left(\zeta_{0}-\zeta\right) p_{i}\right]\left(\pi R_{o}^{2}-\pi R_{i}^{2}\right)
\end{aligned}
$$

where

$$
\begin{aligned}
& \eta_{1(\zeta)}= c_{10} e^{\sqrt{\beta_{1} \zeta}}+c_{10}^{\prime} e^{-\sqrt{\beta_{1} \zeta}}+\left[c_{11} e^{\sqrt{\beta_{1}} \zeta}+c_{11}^{\prime} e^{-\sqrt{\beta_{1} \zeta}}\right. \\
&\left.+\left(A_{1} \zeta e^{\sqrt{\beta_{1} \zeta}}\right)\left(2 \sqrt{\beta_{1}}\right)-\left(B_{1} \zeta e^{-\sqrt{\beta_{1} \zeta}}\right)\left(2 \sqrt{\beta_{1}}\right)\right] \varepsilon, \\
& \eta_{2(\zeta)=} c_{20} e^{\sqrt{\beta_{1} \zeta}}+c_{20}^{\prime} e^{-\sqrt{\beta_{1} \zeta}}+\left[c_{21} e^{\sqrt{\beta_{1} \zeta}}+c_{21}^{\prime} e^{-\sqrt{\beta_{1} \zeta}}\right. \\
&\left.+\left(A_{2} \zeta e^{\sqrt{\beta_{1} \zeta}}\right)\left(2 \sqrt{\beta_{1}}\right)-\left(B_{2} \zeta e^{-\sqrt{\beta_{1} \zeta}}\right)\left(2 \sqrt{\beta_{1}}\right)-\frac{\alpha_{2}}{\beta_{1}}\right] \varepsilon, \\
& \beta_{1}= \beta_{0}^{2}+N^{2}, \\
& \beta_{0}=N \tan \alpha, \\
& \omega=N \varphi+\beta_{0} \zeta,
\end{aligned}
$$

where $\eta_{1}$ and $\eta_{2}$ are parameters of the opening force, $\beta_{0}$ is the groove coefficient, $N$ is the number of spiral grooves, $\varepsilon$ is a small parameter associated with the iterative perturbation, and $\omega$ is the equivalent spiral angle. In addition, the integration constants are as follows: $A_{i}$, where $i=1,2,3 ; B_{i}$, where $i=1,2,3 ; c_{i j}$, where $i=1,2$ and $j=0,1$; and $c_{i j}^{\prime}$, where $i=1,2$ and $j=0,1$.

\subsection{Comparison of the pressure distribution}

According to the Ref. [4], the theoretical pressure distribution of the manuscript is compared with the results available the literature for the SG-DGS. The relevant parameter is listed following: inner diameter is $58.42 \mathrm{~mm}$, the root diameter is $69 \mathrm{~mm}$, outside diameter is $77.78 \mathrm{~mm}$, spiral angle is $15^{\circ}$, the gas film thickness is $2 \mu \mathrm{m}$, the groove number is 12 . The Knudsen number is 0.035 , and the slip effect occurs in the range of $\mathrm{Kn}=0.001-0.1$. The average pressure distributions from a semi analytical model (SAM) of the Ref. [4] and the theoretical model of the current study are shown in Fig. 7. It is shown that the errors between the literature and the theory is small, especially at near the area of the inner radius and outer radius. Additionally, the error is big at near the area of the root radius because of the step and throttling effects between grooved area and grooved-free area. However, these errors can be acceptable because the maximum error of the pressure distribution is about $8 \%$ under different radial direction. Thus, the theoretical model of the current study can provide a good approximate solution for SG-DGS.

\subsection{Calculation of lift-off rotational speed}

The value of the gas film thickness affects the lubrication regime of an SG-DGS. If the gas film thickness is less than the average height of the asperities on both surfaces of the RR and SR, the SG-DGS is in the mixed lubrication regime. Otherwise, the SG-DGS enters the hydrodynamic lubrication regime. Thus, the demarcation point of the gas film thickness is the average asperity height on both surfaces that distinguishes between the mixed and hydrodynamic lubrication regimes. The demarcation point report in Ref. [22] is the minimum contact gas film thickness. In other words, When the minimum gas film thickness is exactly equal to the minimum contact thickness of the gas film, the sealing face is in an opening state; otherwise, the sealing face is in a closed state. The minimum contact thickness of the gas film is equal to 3 times of the overall roughness deviation of the seal surface. The equation for the minimum contact gas film thickness is as follows:

$h_{c}=3 \sigma_{s}=3 \times 1.25 \sqrt{R_{a 1}^{2}+R_{a 2}^{2}}$

where $h_{\mathrm{c}}$ is the minimum contact gas film thickness (lift-off gas film thickness), $\sigma_{\mathrm{s}}$ is Standard deviation combined roughness of both surfaces, and $R_{\mathrm{a} 1}$ and $R_{\mathrm{a} 2}$ are the 


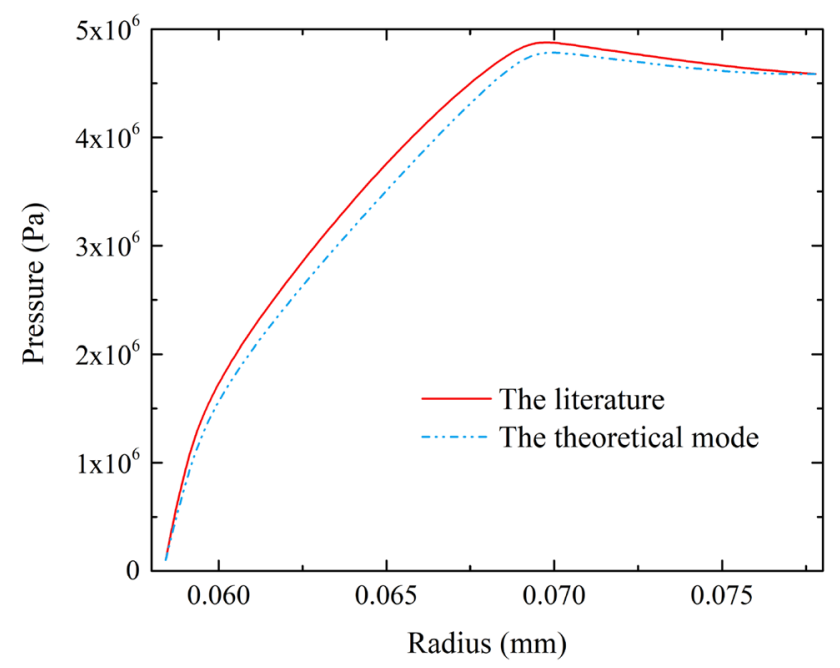

Fig. 7 Comparison of the theoretical results and the literature

arithmetic mean heights of the combined roughness of the two surfaces.

Table 2 lists the physical constants associated with the SG-DGS. The opening force and closing force are calculated according to Eqs. (6) and (30), combined with Tables 1 and 2. Figure 8 illustrates the effect of the rotational speed and gas film thickness on the opening and

Table 2 Physical constants associated with SG-DGS

\begin{tabular}{lll}
\hline Name & Symbol & Value \\
\hline Dynamic viscosity of gas & $\mu$ & $1.8247 \times 10^{-5} \mathrm{~Pa} \mathrm{~s}$ \\
$\begin{array}{l}\text { Gas density } \\
\begin{array}{l}\text { Coefficient of the adjustment in } \\
\text { molecular tangential momentum }\end{array}\end{array}$ & $\sigma_{\mathrm{v}}$ & $1.1885 \mathrm{~kg} / \mathrm{m}^{3}$ \\
\begin{tabular}{l} 
Molecular free path \\
\hline
\end{tabular} & $I$ & 0.3 \\
\hline
\end{tabular}

closing forces along the seal ring faces under spiral angle of $12^{\circ}$. The opening force is changed from 219 to $246.8 \mathrm{~N}$ and a closing force is kept $235 \mathrm{~N}$. With the increase in the rotating speed, more gas is injected into the spiral grooves and is constantly compressed faster in unit time, which results in an increase in the gas film pressure. This result implies that as the rotational speed increases, the higher the load-carrying capacity becomes.

The intersection of the closing and opening forces where it can be concluded that the SG-DGS exists in an equilibrium state. The intersection line is extracted to obtain the value of the rotational speed and gas film thickness at equilibrium, which indicates that the sealing rings are barely separated when the opening force is exactly equal to the closing force. Thus, the intersection line is projected onto axes corresponding to gas film thickness and rotational speed, as shown in Fig. 9. The roughness values of the rotating and stationary rings in Table 1 are incorporated into Eq. (31). The minimum contact gas film thickness of Group 1 is $0.61 \times 10^{-6} \mathrm{~m}$. Furthermore, the value of $0.61 \times 10^{-6} \mathrm{~m}$ is noted in Fig. 9. The lift-off rotational speed corresponds to $276 \mathrm{r} / \mathrm{min}$ for Group 1, and then the lift-off rotational speeds of Group 2, Group 3 and Group 4 can be obtained as well. Finally, the experimental and theoretical lift-off rotational speeds for all specimens are listed in Table 3.

Notably, Table 3 shows that the theoretical lift-off rotating speed varies with the spiral angle. It is illustrated that the spiral angle plays an important role in determining the lift-off rotational speed. It is verified that the effect of the spiral angle on the lift-off rotating speed and the computational model can be used to effectively calculate the lift-off rotational speed of SG-DGS. However, the maximum error is $9.75 \%$. The exclusion of surface friction morphology and contact stress and strain in the calculation could
Fig. 8 Curved surfaces of the opening and closing forces for Group 1

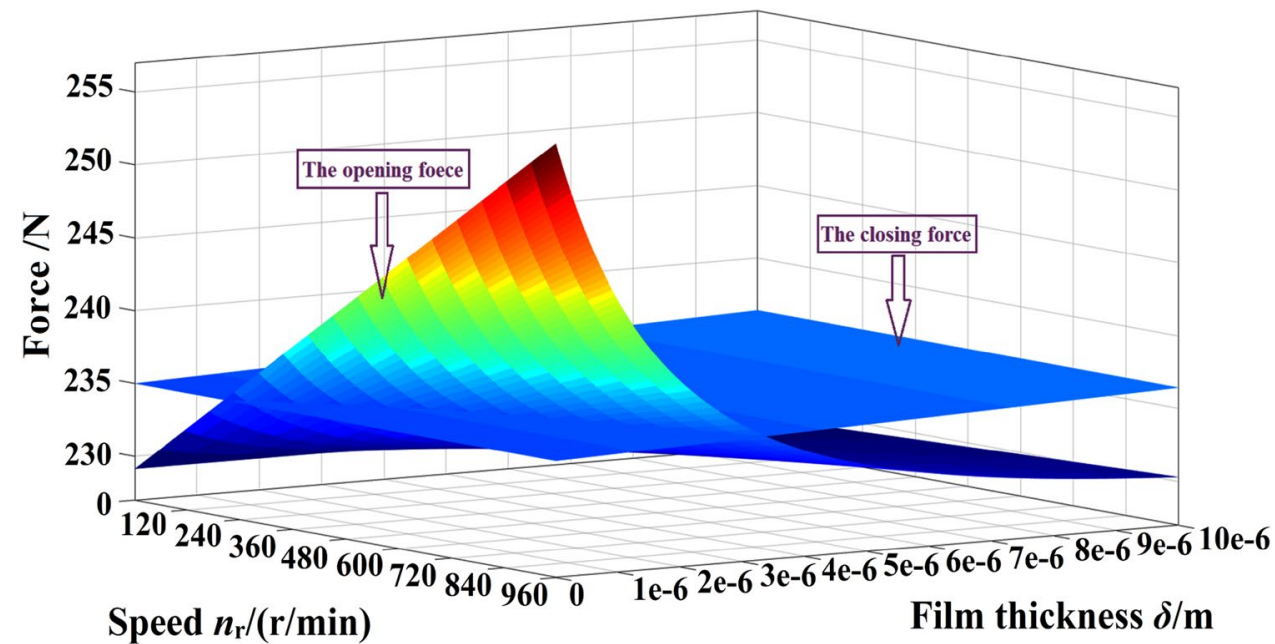




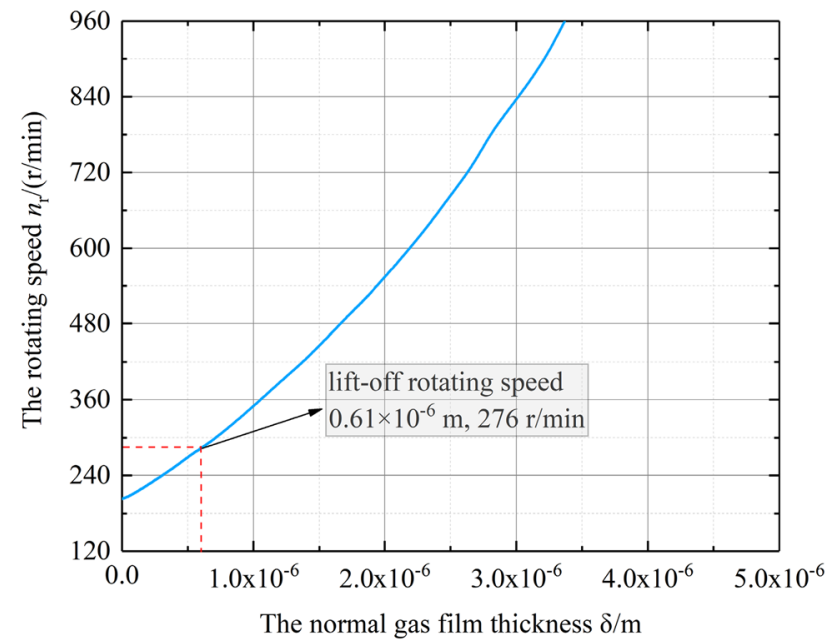

Fig. 9 Lift-off rotating speed for Group 1

Table 3 Experimental and theoretical lift-off rotational speeds for all specimens

\begin{tabular}{llll}
\hline Specimens \# & $\begin{array}{l}\text { Experimental lift-off } \\
\text { speed } n_{\mathrm{r}} /(\mathrm{r} / \mathrm{min})\end{array}$ & $\begin{array}{l}\text { Theoretical lift-off } \\
\text { speed } n_{\mathrm{r}} /(\mathrm{r} / \mathrm{min})\end{array}$ & Error/\% \\
\hline Group 1-12 & 300 & 276 & 8 \\
Group 2-14 & 400 & 361 & 9.75 \\
Group 3-16 & 500 & 462 & 7.6 \\
Group 4-18 & 650 & 622 & 4.3 \\
\hline
\end{tabular}

be a potential reason for the discrepancy. The surface friction morphology and contact stress and strain could result in the deformation of gas film. Hence, a higher lift-off rotational speed is required for start-up. The above factors could be the reason why the calculated lift-off rotational speed is lower than the experimental speed.

\section{Conclusions}

In this study, specimens with $12^{\circ}, 14^{\circ}, 16^{\circ}$ and $18^{\circ}$ spiral angles are processed by a laser marking machine and tested by a face friction machine. Additionally, the tribological behaviors of the specimens are analyzed. Furthermore, a theoretical attempt is performed to calculate and predict the lift-off rotational speed required for startup in the study. The following results are obtained:

1. Transition points from mixed lubrication to full-film lubrication are clearly observed in the experiment.

2. The experiments with different spiral angles indicate that the spiral angle exhibits an important role in determining the lift-off rotational speed of the SG-
DGS, that is, a lower spiral angle can obtain a smaller lift-off rotational speed.

3. The calculation results reveal that the experimental results for the lift-off rotational speed is in good agreement with respect to the starting process. It illustrates that the theoretical model can predict the SGDGS behaviours under start-up and that the structural parameters of the spiral groove change the lift-off rotational speed.

Acknowledgements This paper is based on the research work supported by Ningbo science and technology innovation 2025 major Projects, China (Grant No. 2018B10007) and the National Natural Science Foundation of China (Grant Nos. 51565029 and 51905480).

\section{Compliance with ethical standards}

Conflict of interest The authors declare that they have no conflict of interest.

\section{References}

1. Li W, Yang Y, Sheng DR, Chen JH, Che YQ (2011) Nonlinear dynamic analysis of a rotor/bearing/seal system. J Zhejiang Univ-Sci A (Appl Phys Eng) 12(1):46-55. https://doi.org/10.1631/ jzus.A1000130

2. Zhang GY, Chen GZ, Zhao WG, Yan XT (2017) An experimental test on a cryogenic high-speed hydrodynamic noncontact mechanical seal. Tribol Lett 65(3):65-80. https://doi. org/10.1007/s11249-017-0865-1

3. Miller BA, Green I (2003) Semi-analytical dynamic analysis of spiral-grooved mechanical gas face seal. ASME J Tribol 125(2):403413. https://doi.org/10.1115/1.1510876

4. Xiao KM, Huang WF, Gao WB, Liu XF, Wang YM (2016) A semi-analytical model of spiral-groove face seals: correction and extension. Tribol Trans 59(6):971-982. https://doi.org/10.1080/10402 004.2015.1126876

5. Zhang HJ, Zhu CS, Tang M (2010) Effects of rarefaction on the characteristics of micro gas journal bearings. J Zhejiang Univ Sci A (Appl Phys Eng) 11(1):43-49. https://doi.org/10.1631/jzus. A0900141

6. Ruan B (2000) Finite element analysis of the spiral groove gas face seal at the slow speed and the low pressure conditionsslip flow consideration. Tribol Trans 43(3):411-418. https://doi. org/10.1080/10402000008982357

7. Ma CH, Bai SX, Peng XD (2016) Thermo-hydrodynamic characteristics of spiral groove gas face seals operating at low pressure. Tribol Int 95:44-54. https://doi.org/10.1016/j.tribo int.2015.11.001

8. Ding XX, Lu JJ (2016) Theoretical analysis and experiment on gas film temperature in a spiral groove dry gas seal under high speed and pressure. Int J Heat Mass Transf 96:438-450. https:// doi.org/10.1016/j.ijheatmasstransfer.2016.01.045

9. Green I (2002) A transient dynamic analysis of mechanical seals including asperity contact and face deformation. Tribol Trans 45(3):284-293. https://doi.org/10.1080/10402000208982551

10. Goilkar SS, Hirani H (2010) Parametric study on balance ratio of mechanical face seal in steam environment. Tribol Int 43(56):1180-1185. https://doi.org/10.1016/j.triboint.2009.12.019 
11. Wang J, Jia Q, Yuan XY, Wang SP (2012) Experimental study on friction and wear behaviour of amorphous carbon coatings for mechanical seals in cryogenic environment. Appl Surf Sci 258(24):9531-9535. https://doi.org/10.1016/j.apsus c.2012.05.103

12. Qiu Y, Khonsari MM (2011) Investigation of tribological behaviours of annular rings with spiral groove. Tribol Int 44(12):16101619. https://doi.org/10.1016/j.triboint.2011.05.008

13. Song $X W$, Lin PZ, Liu R, Zhou P (2017) Skin friction reduction characteristics of variable ovoid non-smooth surfaces. J Zhejiang Univ Sci A (Appl Phys Eng) 18(1):59-66. https://doi. org/10.1631/jzus.A1500324

14. Whipple RTP (1951) Theory of the spiral grooved thrust bearing with liquid or gas lubricant[R]. Great Br Atomic Energy Res Establ, Harwell

15. Muijderman EA (1965) Spiral groove bearings[J]. Ind Lubr Tribol 17(1):12-17

16. Huang WF, Lin YB, Gao Z, Fan WJ, Suo SF, Wang YM (2013) An acoustic emission study on the starting and stopping processes of a dry gas seal for pump. Tribol Lett 49(2):379-384. https://doi. org/10.1007/s11249-012-0077-7
17. Salant RF, Cao B (2005) Unsteady analysis of a mechanical seal using Duhamels method. ASME J Tribol 127(3):1017-1023. https ://doi.org/10.1115/1.1924427

18. Liu Y, Liu W, Li YJ, Liu XF, Wang YM (2015) Mechanism of a wavytilt-dam mechanical seal under different working conditions. Tribol Int 90:43-54. https://doi.org/10.1016/j.triboint.2015.03.020

19. Beskok A, Karniadakis GE, Trimmer W (1996) Rarefaction and compressibility effects in gas microflows. J Fluids Eng 118:448456. https://doi.org/10.1115/1.2817779

20. Burgdorfer A (1959) The influence of the molecular mean free path on the performance of hydrodynamic gas lubricated bearing. ASME J Basic Eng 81(1):94-100

21. Ausman JS (1961) An improved analytical solution for self-acting, gas-lubricated journal bearings of finite length. J Fluids Eng 83(2):188-192. https://doi.org/10.1115/1.3658920

22. Lebeck AO (1991) Principle and design of mechanical face seals. Wiley, New York, pp 107-185

Publisher's Note Springer Nature remains neutral with regard to jurisdictional claims in published maps and institutional affiliations. 